\title{
RASS-SDSS galaxy cluster survey
}

\section{On the cluster mass-to-light ratio and the halo occupation distribution}

\author{
P. Popesso ${ }^{1}$, A. Biviano ${ }^{2}$, H. Böhringer ${ }^{3}$, and M. Romaniello ${ }^{1}$ \\ 1 European Southern Observatory, Karl Scharzschild Strasse 2, 85748 Garching, Germany \\ e-mail: ppopesso@eso.org \\ 2 INAF - Osservatorio Astronomico di Trieste, via G.B. Tiepolo 11, 34131 Trieste, Italy \\ 3 Max-Planck-Institut für Extraterrestrische Physik, 85748 Garching, Germany
}

Received 16 December 2005 / Accepted 8 June 2006

ABSTRACT

\begin{abstract}
Aims. We explore the mass-to-light ratio in galaxy clusters and its relation to the cluster mass.
Methods. We study the relations among the optical luminosity $\left(L_{\mathrm{op}}\right)$, the cluster mass $\left(M_{200}\right)$ and the number of cluster galaxies within $r_{200}\left(N_{\mathrm{gal}}\right)$ in a sample of 217 galaxy clusters with confirmed 3D overdensity. We correct for projection effect, by determining the galaxy surface number density profile in our cluster sample. This is best fitted by a cored King profile in low and intermediate mass systems. The core radius decreases with cluster mass, and, for the highest mass clusters, the profile is better represented by a generalized King profile or a cuspy Navarro, Frenk \& White profile.

Results. We find a very tight proportionality between $L_{\mathrm{op}}$ and $N_{\text {gal }}$, which, in turn, links the cluster mass-to-light ratio to the Halo Occupation Distribution $N_{\text {gal }}$ vs. $M_{200}$. After correcting for projection effects, the slope of the $L_{\mathrm{op}}-M_{200}$ and $N_{\mathrm{gal}}-M_{200}$ relations is found to be $0.92 \pm 0.03$, close, but still significantly less than unity. We show that the non-linearity of these relations cannot be explained by variations of the galaxy luminosity distributions and of the galaxy $M / L$ with the cluster mass.

Conclusions. We suggest that the nonlinear relation between number of galaxies and cluster mass reflects an underlying nonlinear relation between number of subhaloes and halo mass.
\end{abstract}

Key words. galaxies: clusters: general - galaxies: luminosity function, mass function

\section{Introduction}

Clusters of galaxies are the most massive gravitationally bound systems in the universe. The cluster mass function and its evolution provide constraints on the evolution of large-scale structure and important cosmological parameters such as $\Omega_{\mathrm{m}}$ and $\sigma_{8}$. Cluster mass-to-light ratios $(M / L$ hereafter $)$ provide one of the most robust determination of $\Omega_{\mathrm{m}}$ in connection with the observed luminosity density in the Universe via the Oort (1958) method. In this method, a fundamental assumption is that the average $M / L$ of clusters is a fair representation of the universal value. For this reason, many works have focused on the dependence of the cluster $M / L$ on the mass of the systems. In general, $M / L$ has been found to increase with the cluster mass. Assuming a power-law relation $M / L \propto M^{\alpha}$, and adopting the usual scaling relations between mass and X-ray temperature or velocity dispersion, when needed, most authors have found $\alpha$ in the range 0.2-0.4, in both optical and near-infrared bands, and over a large mass range (Adami et al. 1998a; Bahcall \& Comerford 2002; Girardi et al. 2002; Lin et al. 2003, 2004; Rines et al. 2004; Ramella et al. 2004; see however Kochanek et al. 2003 , for a discordant result). Why does the cluster $M / L$ increase with the mass? Based on the results of numerical simulations, Bahcall \& Comerford (2002) have proposed that the trend of $M / L$ with mass is caused by the stellar populations of galaxies in more massive systems being older than the stellar populations of galaxies in less massive systems. In this scenario, the slope of the $M / L-M$ relation should be steeper in the $B$ and $V$ bands, dominated by the young stellar populations, than at longer wavelengths, eventually becoming flat in the infrared $K$ band, dominated by the light of the old stellar population. Such a scenario is not consistent with the results of the semi-analytical modeling of Kauffmann et al. (1999), where the $M / L$ is predicted to increase with mass with approximately the same slope in the $B$ and $I$ band. Also observationally, the slope of the $M / L-M$ relation is found to be the same in different bands, the $B$-band (Girardi et al. 2002) the $V$-band (Bahcall \& Comerford 2002), the $R$-band (Adami et al. 1998a; Popesso et al. 2005b, 2007) and the $K$-band (Lin et al. 2003, 2004; Rines et al. 2004; Ramella et al. 2004). An alternative interpretation of the increasing $M / L$ with system mass is provided by Springel \& Hernquist (2003). They analyze the star formation efficiency within halos extracted from cosmological simulations, with masses in the range $10^{8}-$ $10^{15} M_{\odot}$, and find that the integrated star formation efficiency decreases with increasing halo mass by a factor 5-10 over the cluster mass range. This scenario is investigated by Lin et al. (2003), who convert the 2MASS $K$-band cluster luminosities into cluster stellar masses. They find that the fraction of mass in stars is a decreasing function of the cluster mass $\left(M_{\mathrm{star}} / M_{\mathrm{tot}} \propto M_{\mathrm{tot}}^{-0.26}\right)$.

In this paper we address the above issues by studying $M / L$ for a sample of 217 clusters, which span the entire cluster mass range. In particular, we study the relations among the cluster optical luminosity $L_{\mathrm{op}}$, the mass $M_{200}$, and the number of cluster galaxies $N_{\text {gal }}$, within the virial radius $r_{200}$. We find a very tight relation between $L_{\mathrm{op}}$ and $N_{\mathrm{gal}}$, which links the $L_{\mathrm{op}}-M_{200}$ relation (and therefore, the cluster $M / L$ ), to the Halo Occupation Distribution (HOD hereafter) $N_{\text {gal }}-M_{200}$. The HOD is a powerful tool for describing galaxy bias and modelling galaxy 
clustering (e.g. Ma \& Fry 2000; Peacock \& smith 2000; Seljak 2000; Scoccimarro et al. 2001; Berlind \& Weinberg 2002). It characterizes the bias between galaxies and mass in terms of the probability distribution $P(N \mid M)$ that a halo of virial mass $M$ contains $N$ galaxies of a given type, together with relative spatial and velocity distributions of galaxies and dark matter within halos. The HOD is a fundamental prediction of galaxy formation theory (e.g. Kauffmann et al. 1997, 1999; White et al. 2001; Yoshikawa et al. 2001; Berlind et al. 2003; Kravtsov et al. 2004; Zheng et al. 2005) and it can be extremely useful to compare the observational results with the theoretical models.

This paper is organized as follows. In Sect. 2 we describe our dataset. In Sect. 3 we describe the methods we use to calculate several cluster properties, like the characteristic radius, the virial mass, the optical luminosity, and the number density profile of cluster galaxies. In Sect. 4 we analyze the $L_{\text {op }}-M_{200}$ and the $N_{\text {gal }}-M_{200}$ relations, and find that the number of galaxies per given halo mass decreases as the halo mass increases. In Sect. 5 we seek a physical explanation of this trend by comparing our results with theoretical predictions. In Sect. 6 we provide our conclusions.

Throughout this paper, we use $H_{0}=70 \mathrm{~km} \mathrm{~s}^{-1} \mathrm{Mpc}^{-1}$ in a flat cosmology with $\Omega_{0}=0.3$ and $\Omega_{\Lambda}=0.7$ (e.g. Tegmark et al. 2004).

\section{The data}

The optical data used in this paper are taken from the Sloan Digital Sky Survey (SDSS, Fukugita 1996; Gunn et al. 1998; Lupton et al. 1999; York et al. 2000; Hogg et al. 2001; Eisenstein et al. 2001; Smith et al. 2002; Strauss et al. 2002; Stoughton et al. 2002; Blanton et al. 2003, and Abazajian et al. 2003). The SDSS consists of an imaging survey of $\pi$ steradians of the northern sky in the five passbands $u, g, r, i, z$, in the entire optical range. The imaging survey is taken in drift-scan mode. The imaging data are processed with a photometric pipeline (PHOTO, Lupton et al. 2001) specially written for the SDSS data. For each cluster we defined a photometric galaxy catalog as described in Sect. 3 of Popesso et al. (2004; see also Yasuda et al. 2001). For the analysis in this paper we use only SDSS Model magnitudes. The discussion about completeness limits in magnitude and surface brightness of the SDSS galaxy photometric sample can be found in Popesso et al. (2005a,b, Papers II and IV of this series).

The spectroscopic component of the survey is carried out using two fiber-fed double spectrographs, covering the wavelength range 3800-9200 $\AA$, over 4098 pixels. They have a resolution $\Delta \lambda / \lambda$ varying between 1850 and 2200 , and together they are fed by 640 fibers, each with an entrance diameter of 3 arcsec. The fibers are manually plugged into plates inserted into the focal plane; the mapping of fibers to plates is carried out by a tiling algorithm (Blanton et al. 2003) that optimizes observing efficiency in the presence of large-scale structure.

\subsection{The cluster sample}

In this paper we use a combined sample of X-ray selected galaxy clusters and optically selected systems. The X-ray selected clusters are taken from the RASS-SDSS galaxy cluster catalog of Popesso et al. (2005b, hereafter Paper III), which comprises 130 systems selected mainly from the REFLEX and NORAS X-ray cluster catalogs. The optically selected clusters are taken from Popesso et al. (2007), who selected a subsample of 130 Abell clusters with confirmed 3-dimensional galaxy overdensity in the

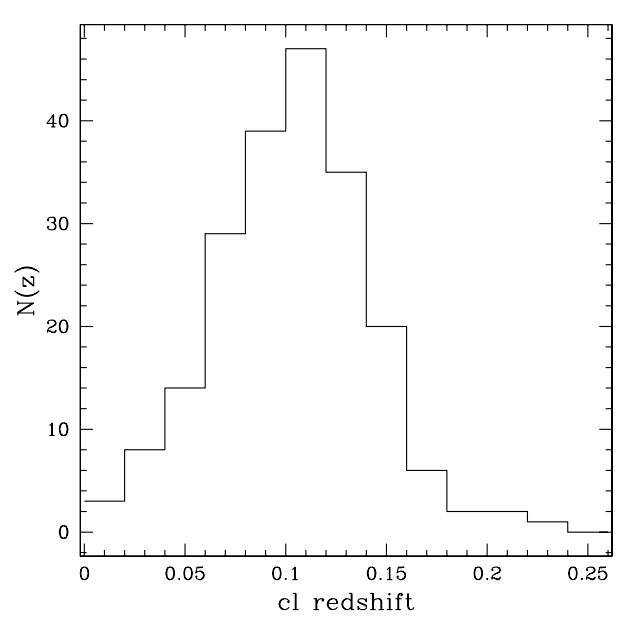

Fig. 1. Redshift distribution of the cluster sample used in this paper.

third release of the SDSS galaxy spectroscopic catalog. The two samples overlap with 43 clusters. The combined sample with the exclusion of the double detections comprises 217 clusters and covers the entire range of masses and X-ray/optical luminosities, from very low-mass and X-ray/optical faint groups $\left(10^{13} M_{\odot}\right)$ to very massive and X-ray/optical bright clusters $\left(5 \times 10^{15} M_{\odot}\right)$. The cluster sample comprises only nearby systems at the mean redshift of 0.1 . The redshift distribution of the cluster sample is shown in Fig. 1.

\section{The cluster properties}

In this section we explain the methods used to calculate the cluster properties as the characteristic radius, the virial mass, the optical luminosity and the parameters of the radial profile of the cluster galaxies.

\subsection{Characteristic radii and masses}

Here we describe the methods by which we measure the characteristic cluster radii $r_{200}$ and mass $M_{200} . r_{200}$ and $M_{200}$ are the radius and the mass, respectively, where the mass density of the system is 200 times the critical density of the Universe and it is considered as a robust measure of the virial radius of the cluster.

Estimates of cluster velocity dispersion, mass, and characteristic radius requires knowledge of the redshifts of its member galaxies. We have used the redshifts provided in the SDSS spectroscopic catalog.

Cluster members are selected following the method of Adami et al. (1998a) or Girardi et al. (1993), depending on whether the mean cluster redshift $z_{\text {cluster }}$ is known in advance (from previous studies) or not, respectively. Girardi et al. (1993) method requires in fact that a preliminary cut be done in the line-of-sight velocity space, $\pm 4000 \mathrm{~km} \mathrm{~s}^{-1}$ around $c z_{\text {cluster }}$, before searching for significant weighted-gaps in the velocity distribution. On the other hand, the density-gap technique of Adami et al. does not require such a preliminary cut. If $z_{\text {cluster }}$ is known already, we select among the groups identified by the gapping technique that one closest in velocity space to $c z_{\text {cluster }}$, otherwise we select the most populated one. After the initial group selection, we apply the interloper-removal method of Katgert et al. (2004; see Appendix A in that paper for more details) on the remaining galaxies, using the X-ray center when available, or else 
the position of the brightest cluster galaxy on the cluster colourmagnitude sequence.

The virial analysis (see, e.g., Girardi et al. 1998) is then performed on the clusters with at least 10 member galaxies. The velocity dispersion is computed on the cluster members, using the biweight estimator (Beers et al. 1990). The virial masses are corrected for the surface pressure term (The \& White 1986) by adopting a profile of Navarro et al. (1996, 1997, NFW hereafter) with a concentration parameter, $c$, that depends on the initial estimate of the cluster virial mass itself. The $c$-mass relation is given by $c=4 \times\left(M / M_{\mathrm{KBM}}\right)^{-0.102}$ where the slope of the relation is taken from Dolag et al. (2004), and the normalization $M_{\mathrm{KBM}} \simeq 2 \times 10^{15} M_{\odot}$ from Katgert et al. (2004). The clusters in our sample span a range $c \simeq 3-6$.

Correction for the surface pressure term requires knowledge of the $r_{200}$ radius, for which we adopt the Carlberg et al. (1997) definition (see Eq. (8) in that paper) as a first guess. After the virial mass is corrected for the surface pressure term, we refine our $r_{200}$ estimate using the virial mass density itself. Let $M_{\mathrm{vir}}$ be the virial mass (corrected for the surface term) contained in a volume of radius equal to a chosen observational aperture, $r_{\mathrm{ap}}$. The radius $r_{200}$ is then given by:

$r_{200} \equiv r_{\text {ap }}\left[\rho_{\text {vir }} /\left(200 \rho_{\mathrm{c}}\right)\right]^{1 / 2.4}$

where $\rho_{\text {vir }} \equiv 3 M_{\text {vir }} /\left(4 \pi r_{\text {ap }}^{3}\right)$ and $\rho_{\mathrm{c}}(z)$ is the critical density at redshift $z$ in the adopted cosmology. The exponent in Eq. (1) is the one that describes the average cluster mass density profile near $r_{200}$, as estimated by Katgert et al. (2004) for an ensemble of 59 rich clusters.

A NFW profile is used to interpolate (or, in a few cases, extrapolate) the virial mass $M_{\text {vir }}$ from $r_{\text {ap }}$ to $r_{200}$, yielding $M_{200}$. As before, we scale the concentration parameter of the used NFW profile according to a preliminary estimate of the mass of the system. From $M_{200}$ the final estimate of $r_{200}$ is obtained, using the definition of $M_{200}$ itself.

\subsection{Optical luminosities}

The total optical luminosity of a cluster has to be computed after the subtraction of the foreground and background galaxy contamination. We consider two different approaches to the statistical subtraction of the galaxy background. We compute the local background number counts in an annulus around the cluster and a global background number counts from the mean of the magnitude number counts determined in five different SDSS sky regions, randomly chosen, each with an area of $30 \mathrm{deg}^{2}$. In our analysis we show the results obtained using the optical luminosity estimated with the second method. The optical luminosity is then computed within $r_{200}$ following the prescription of Popesso et al. (2004). The reader is referred to that paper for a detailed discussion about the comparison between optical luminosities calculated with different methods. To avoid selection effects due to the slightly different redshifts of the clusters, the optical luminosity has been calculated in the same absolute magnitude range for all the clusters. The adopted range has been varied to check the robustness of the results of the regression analyses.

\subsection{Number density profiles and projection effects}

The observed cluster optical luminosity, $L_{\mathrm{op}}$, is contributed not only by galaxies within the virial sphere of radius $r_{200}$, but also by galaxies outside the virial sphere yet within the cylinder of

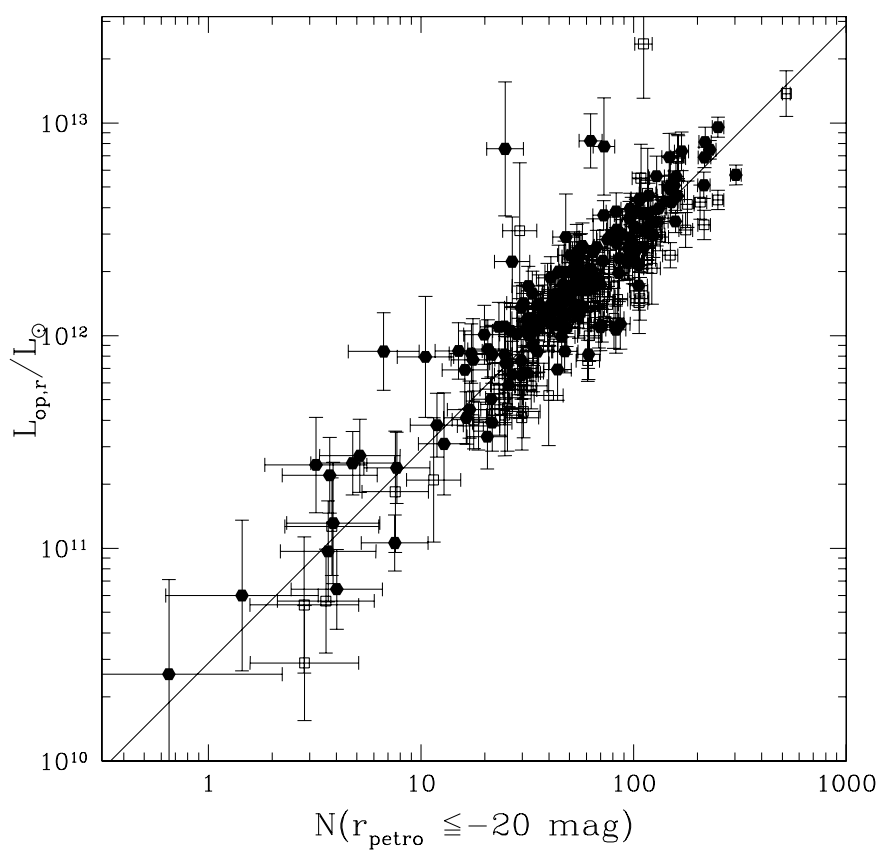

Fig. 2. Relation between the optical luminosity calculated in the SDSS $r$ band within $r_{200}$ and the number of cluster galaxies contributing to $L_{\mathrm{op}}$. The solid line is the best fit line with slope $1.00 \pm 0.03$.

same radius. It is therefore necessary to correct the observed optical luminosity for the contribution of cluster galaxies outside the virial sphere (the following analysis is based on photometric data and the field galaxies contribution is removed statistically as described in the previous section).

Figure 2 shows the proportionality between the cluster $r$-band optical luminosity within $r_{200}$ and the number of cluster galaxies $\left(N_{\mathrm{gal}}\right)$, contributing to the luminosity itself, i.e. the background-subtracted galaxy counts within the same radius, down to the magnitude limit used to estimate $L_{\mathrm{op}}$. Because of the strict proportionality between $L_{\mathrm{op}}$ and $N_{\mathrm{gal}}$, we can use the ratio between the number of cluster galaxies within the cylindrical volume and the number of galaxies within the virial sphere, to correct the observed $L_{\mathrm{op}}$ for the contribution of cluster galaxies outside the virial sphere. In order to estimate this ratio, we build the surface number density profiles of our clusters, and fit them with two widely-used analytical functions, the King (1962) cored profile, and the NFW cuspy profile. The 3D and projected King profiles are given by, respectively:

$\rho(r)=\frac{n_{0}}{\left(1+\left(r / r_{\mathrm{c}}\right)^{2}\right)^{3 / 2}}$

and:

$\sigma(b)=\frac{\sigma_{0}}{\left(1+\left(b / r_{\mathrm{c}}\right)^{2}\right)}$

where $r_{\mathrm{c}}$ is the core radius and $\sigma_{0}=2 n_{0} r_{\mathrm{c}}$ is the normalization (see also Sarazin 1980). The NFW profile in 3D is given by:

$\rho(r)=\frac{\delta_{0}}{r / r_{\mathrm{s}}\left(1+\left(r / r_{\mathrm{s}}\right)^{2}\right)}$

where $r_{\mathrm{s}}$ is the characteristic radius $\left(r_{\mathrm{s}}=r_{200} / c\right.$ with $c$ the concentration parameter) and $\delta_{0}$ is the normalization. The projected surface density profile is then obtained from an integration of the three-dimensional profile (see Bartelmann et al. 1996). 


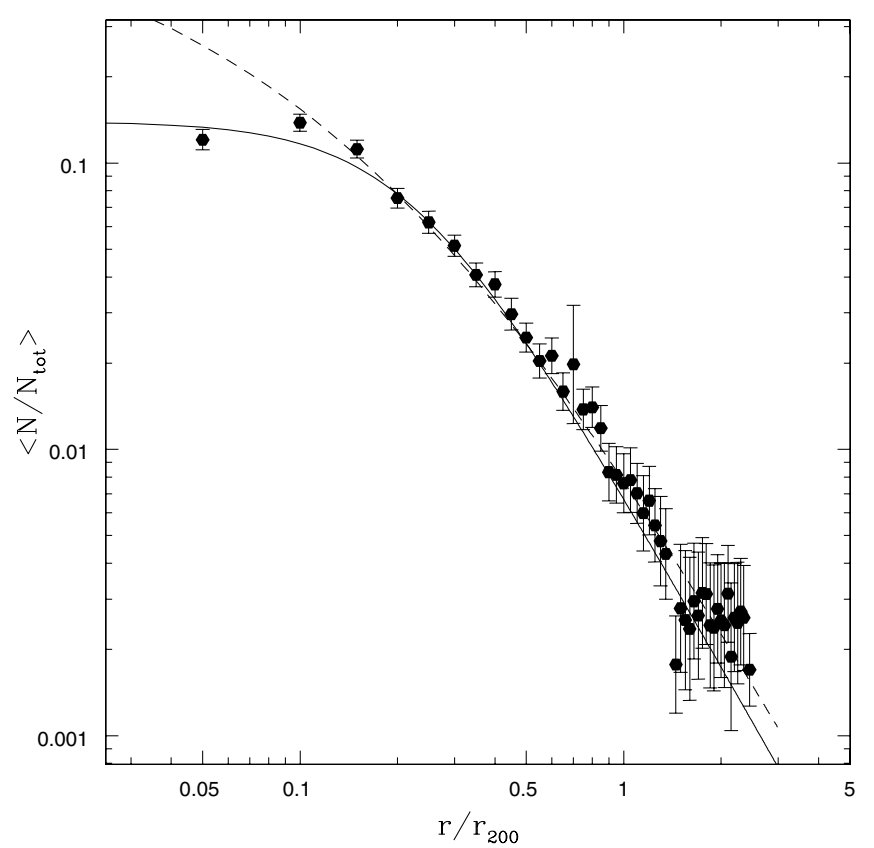

Fig. 3. The stacked mean surface number density profile of all the cluster galaxies with magnitude $r<-18.5$. The solid curve is the best fit King profile, the dashed curve is the best fit NFW profile.

As a first step we explore the the mean surface density galaxy distribution within our cluster sample, by stacking the projected galaxy distributions of the individual systems. Note that in this analysis we only consider the clusters with available X-ray centers, in order to reduce possible mis-centering when adopting the positions of brightest cluster galaxies as cluster centers (not all brightest cluster galaxies lie at centers of their parent clusters, see, e.g., Lin \& Mohr 2004). The clustercentric distances are rescaled to the cluster $r_{200}$ before the stacking. The cluster galaxy distributions are normalized to the total number of galaxies within $r_{200}$, after subtraction of the mean background galaxy density, evaluated within the $2.5-3.5 \times r_{200}$ annulus. Figure 3 shows the stacked surface density profile of all the 217 clusters. The best fit is given by a King profile with core radius $r_{\mathrm{c}} / r_{200}=0.224 \pm 0.005$, while a NFW profile provides a poor fit near the centre. We then split our sample of clusters in 6 mass bins: $M_{200} / 10^{14} M_{\odot} \leq 1,1<M_{200} / 10^{14} M_{\odot} \leq 3$, $3<M_{200} / 10^{14} M_{\odot} \leq 7,7<M_{200} / 10^{14} M_{\odot} \leq 10$, $10<M_{200} / 10^{14} M_{\odot} \leq 30$, and $M_{200} / 10^{14} M_{\odot}>30$. Each bin contains at least 10 clusters.

Figure 4 shows the surface density profiles in each cluster mass bin. The solid line in each plot shows the King profile that provides the best-fit to the surface density profile of all galaxies in all clusters, already shown in Fig. 3. The dashed line in each panel is the best-fit King profile for the surface density profile of each cluster mass bin. The NFW profiles provide poor fits for most cluster mass bins, and are not plotted. From Fig. 4 one can clearly see how the cluster galaxy distribution changes with cluster mass. The surface density profiles become steeper near the centre as the cluster mass increases. Note that the surface number density profile in the low mass bin $\left(M_{200} / 10^{14} M_{\odot} \leq 1\right)$ is not completely consistent with a King profile since it shows a deficit of galaxies near the center. The core radius is quite large, $r_{\mathrm{c}} / r_{200}=0.40 \pm 0.08$. The core radius becomes smaller as the cluster mass increases, and it is $r_{\mathrm{c}} / r_{200}=0.16 \pm 0.01$ for clusters in the mass interval $7<M_{200} / 10^{14} M_{\odot} \leq 10$. In the last two mass bins, the galaxy distributions become so concentrated that the simple King profile no longer provides a good fit, and a generalized King profile is needed, of the form:

$\sigma(b)=\frac{\sigma_{0}}{\left(1+\left(b / r_{\mathrm{c}}\right)^{2}\right)^{\beta}}$.

The dotted lines in the panels of Fig. 4 corresponding to the highest cluster mass bins, show the best fit given by the generalized King profile, where $\beta=0.91 \pm 0.01$. Finally, in the highest mass bin $\left(M_{200}>3 \times 10^{15}\right)$ the projected NFW profile also provides a good fit to the galaxy distributions. This is shown in Fig. 5, where the best-fit generalized King profile is shown as a solid curve, and the best-fit NFW profile is shown as a dashed curve. In this case, the best-fit value of the NFW concentration parameter is $c=4.2 \pm 0.3$, and is consistent with the value found for the dark matter distribution in similarly massive clusters (e.g. Biviano \& Girardi 2003; Katgert et al. 2004).

Lin et al. (2004) perform the same analysis on a smaller sample of 93 X-ray selected clusters observed in the 2 MASS all sky survey. Their conclusion is that the surface density profile of cluster galaxies is consistent with a NFW profile with concentration parameter $2.90 \pm 0.22$. They study the galaxy distribution in 2 mass bins with mean mass $\left\langle M_{500}\right\rangle=7.9 \times 10^{13} M_{\odot}$ for the groups, and $\left\langle M_{500}\right\rangle=5.3 \times 10^{14} M_{\odot}$, for the massive clusters, and claim that the spatial profiles are consistent with the mean profile in both mass bins. However, by fitting their data (taken from Fig. 8 of Lin et al. 2004) with both a King, a generalized King, and a NFW profile, we find that a King profile provides the best fit, in agreement with our findings.

Our results are further supported by the analysis of the surface brightness profile of our clusters. Figure 6 shows the composite surface brightness profile of two cluster subsamples: the low-mass systems with $M_{200} \leq 10^{14} M_{\odot}$ and the high-mass clusters with $M_{200}>3 \times 10^{15} M_{\odot}$. The profile of the low-mass objects displays a core, and is less centrally concentrated than that of the high-mass clusters which is in fact rather cuspy. As expected, due to the presence of the Brightest Cluster Galaxies at the center of the systems, the luminosity profiles are generally more cuspy than the density profiles in the same cluster mass bins (Adami et al. 2001).

In stacking clusters, we have assumed circularity, because the number of galaxies per cluster is generally too small to allow a precise determination of individual cluster shapes and orientations. Adami et al. (1998b) have shown that enforcing circularity could create a central artificial cusp in the number density profile of the stacked cluster. However, lower mass clusters are more elongated than higher mass clusters (see Fasano et al. 1993; de Theije et al. 1995; Plionis et al. 2004), so the effect of assuming circularity should lead to more cuspy density profiles for lower mass clusters, which is opposite to what we find. Indeed, the effect reported by Adami et al. does not seem to be strong enough to account for the differences seen in the density profiles of the stacked clusters of different masses (compare Fig. 4 with Fig. 7 in Adami et al. 1998b).

Hence we conclude that there is a significant variation of the number density and luminosity density profiles of clusters, as a function of cluster mass, with higher mass clusters displaying more concentrated profiles. As a consequence, also the correction needed to convert the number of galaxies contained in the cylindrical volume to that in the virial sphere depends on the cluster mass. Using the volume and the surface density King profile given in Eqs. (2) and (3), respectively, we estimate that 

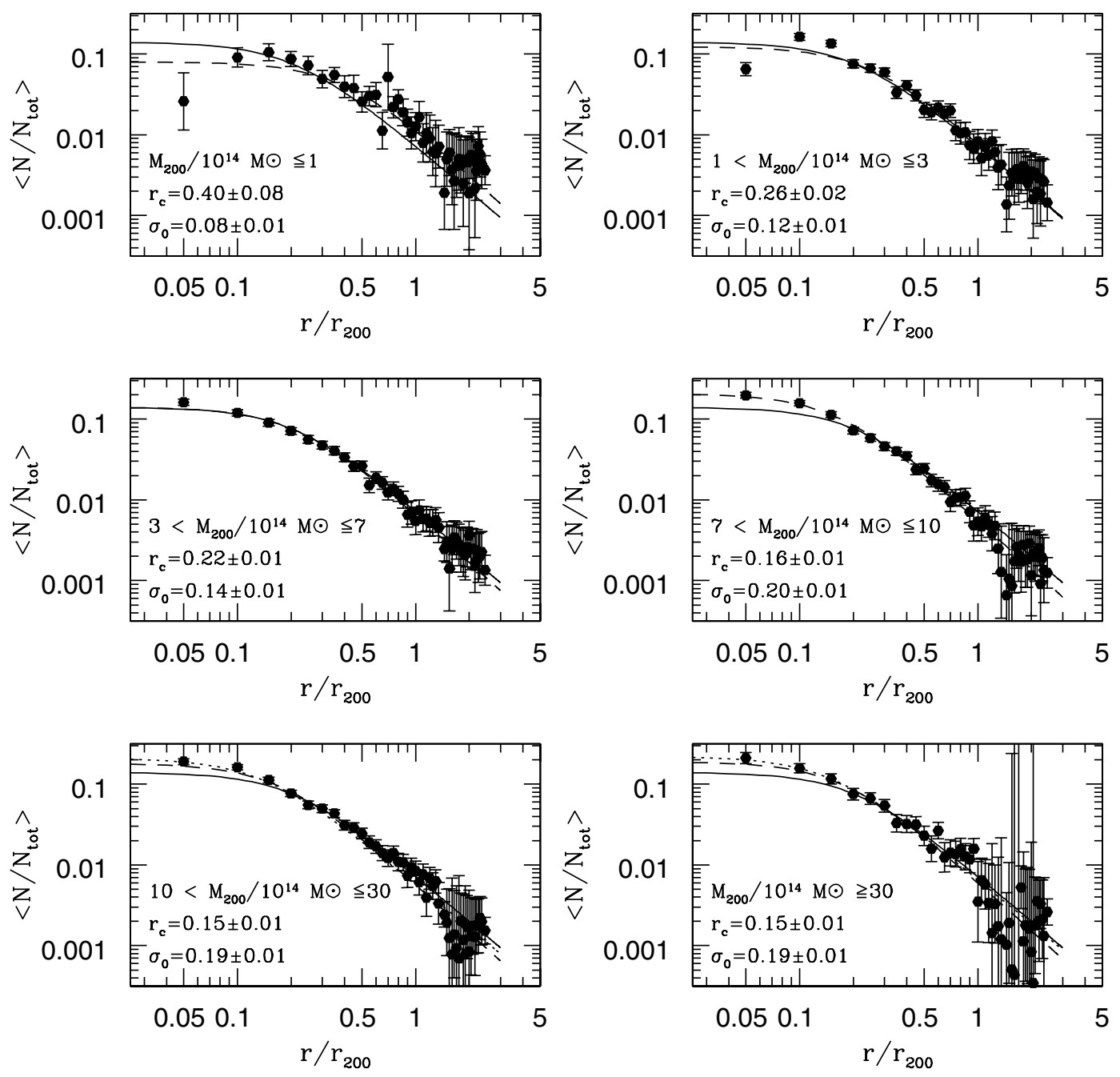

Fig. 4. The stacked surface number density profiles of clusters in different cluster mass bins. The individual cluster profiles are obtained by considering all the galaxies with $r_{\text {petro }}<-18.5$. The dashed curve in each panel is the King profile that provides the best-fit to the surface density profile shown in that same panel, while the solid curve is the King profile that provides the best-fit to the mean stacked surface density profile (same as the solid line in Fig. 3). The dotted line in the bottom panels (corresponding to the highest-mass bin clusters) is the best fit provided by a generalized King profile.

the ratio between the number of galaxies in the virial sphere of radius $r_{200}$ and the number of galaxies actually observed in the cylinder of same radius is $0.69-0.76$ for clusters in the lowestmass bin, $0.78-0.80$ for clusters in the $1-3 \times 10^{14} M_{\odot}$ mass bin, 0.81 in the $3-7 \times 10^{14} M_{\odot}$ mass bin, and 0.85 in the highest mass bins.

We performed the same analysis separately for the red (earlytype) and blue (late-type) cluster galaxy populations. The colour separation between the two population is based on the SDSS galaxy color $u-r$ (Strateva et al. 2001; Popesso et al. 2006). For both the red and the blue galaxy populations, the core radius of the best-fit King profile monotonically decreases from the lowmass systems to the more massive clusters (see Figs. 7 and 8).

Since there is a significant mass-dependence of the number density profiles, a mass-dependent deprojection correction needs to be applied to the observed values of $L_{\mathrm{op}}$. In the following, we only consider the deprojection-corrected values of $L_{\mathrm{op}}$, obtained by adopting the correction factors per mass bin derived above.

\section{The $L_{o p}-M_{200}$ and the $N_{\text {gal }}-M_{200}$ relations}

In Fig. 9 we show the r-band $L_{\mathrm{op}}-M_{200}$ relation after correcting $L_{\mathrm{op}}$ for the projection effects (see Sect. 3.3). The solid line in the figure is the best-fit linear regression in logarithmic space, and the dotted line is the best-fit we would have obtained had $L_{\mathrm{op}}$ not been corrected for the projection effects. The best-fit relation for the corrected $L_{\mathrm{op}}$ is:

$$
L_{\mathrm{op}} /\left(10^{12} L_{\odot}\right)=10^{-0.29 \pm 0.03}\left(M_{200} /\left(10^{14} M_{\odot}\right)\right)^{0.92 \pm 0.03}
$$

The slope of this relation is steeper than the slope of the uncorrected relation, which is $0.85 \pm 0.03$. The two values are anyhow marginally consistent within $2.5 \sigma$. As a consequence, also the $M / L-M$ relation is flatter. The slope of the corrected relation is $0.18 \pm 0.04$ instead of $0.27 \pm 0.04$ for the uncorrected relation. Remarkably, we find that the slopes of the best-fit $L-M$ and $M / L-M$ relations do not depend on the chosen photometric SDSS band. 


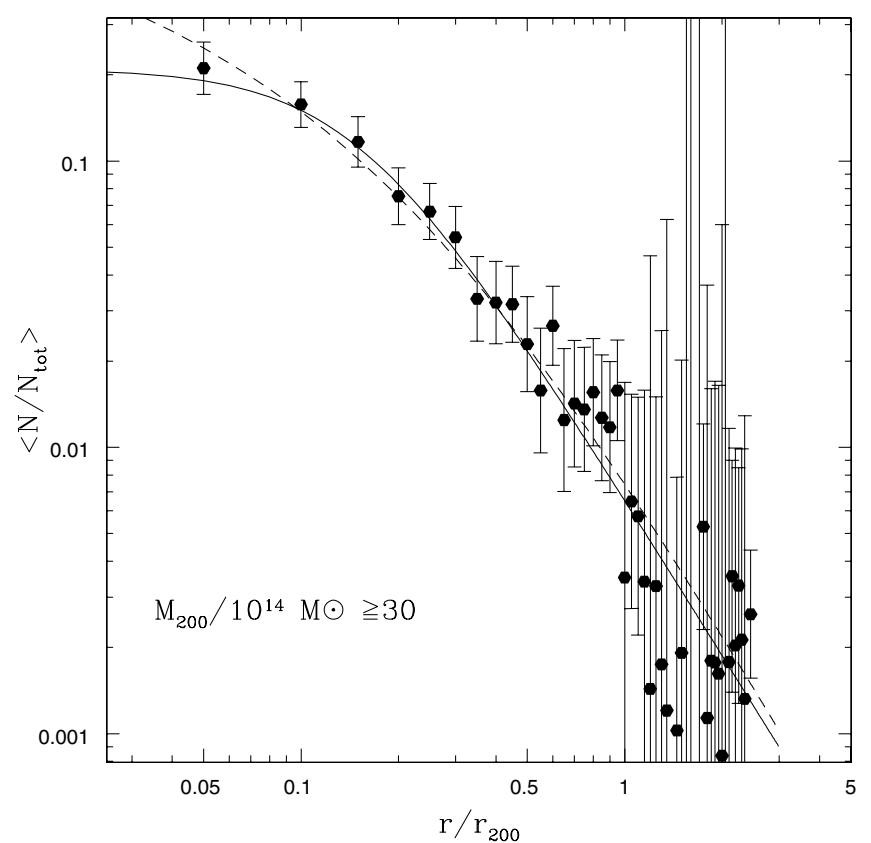

Fig. 5. The surface number density profile of all the cluster galaxies with $r<-18.5$ in the highest of our considered cluster mass bins. The solid curve is the best fit provided by the generalized King profile. The dashed curve is the best fit provided by the NFW profile. Both profiles are consistent with the data.

Due to the strict proportionality observed between the cluster optical luminosity and the number of cluster galaxies (see Fig. 2), it is clear that the $L_{\mathrm{op}}-M_{200}$ relation is strictly connected to the $N_{\text {gal }}-M_{200}$ relation. In other words, the cluster mass-tolight ratio $M / L$ is strictly related to the Halo Occupation Number $\gamma$ of the Halo Occupation Distribution (HOD) $N \propto M^{\gamma}$. It is then useful to study the cluster $M / L$ in terms of the HOD since this allows an easier comparison with the predictions of models of structure formation.

We study the HOD with two approaches. First we use the $N_{\text {gal }}$ calculated using the photometric data $\left(N_{\text {phot }}\right)$, by summing the background-subtracted cluster number counts used to calculate $L_{\mathrm{op}}$. As a second approach we estimate the number of spectroscopically-confirmed cluster members $\left(N_{\text {spec }}\right)$. Both estimates are corrected for projection effects in the same way as we did for $L_{\mathrm{op}}$. Both $N_{\text {phot }}$ and $N_{\text {spec }}$ are computed down to the same absolute magnitude, in order to allow comparison of the two estimates. The SDSS spectroscopic and photometric catalogs have two different apparent magnitude limits ( $r=17.77$ for the spectroscopic catalog and $r \sim 21 \mathrm{mag}$ for the photometric one). We apply an absolute magnitude cut of $M_{r} \leq-20$, which allows us to sample the cluster luminosity function (LF hereafter) down to $M^{*}+2$ (Popesso et al. 2005a). With such a cut, $N_{\text {spec }}$ can be measured for a significant fraction of our cluster sample, those 90 clusters for which $M_{r} \leq-20$ is brighter than the apparent magnitude limit of $r=17.77$.

In Fig. 10 we show the $N_{\mathrm{gal}}-M_{200}$ relation, using $N_{\mathrm{gal}} \equiv$ $N_{\text {phot }}$. We also plot the best fit relations

$N_{\text {gal }}=10^{-11.60 \pm 0.59}\left(M_{200} / M_{\odot}\right)^{0.91 \pm 0.04}$

for $N_{\text {gal }} \equiv N_{\text {phot }}$, and

$N_{\text {gal }}=10^{-11.43 \pm 0.76}\left(M_{200} / M_{\odot}\right)^{0.89 \pm 0.05}$

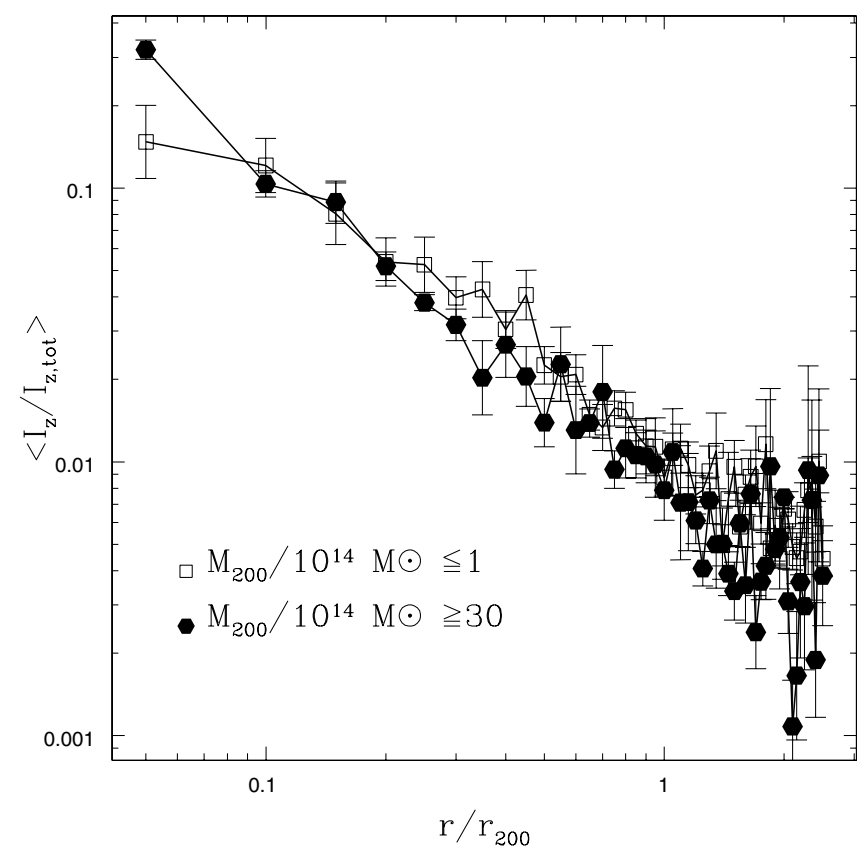

Fig. 6. Composite surface brightness profile of two cluster samples: the low mass systems at $M_{200} \leq 10^{14} M_{\odot}$ (empty squares) and the massive clusters at $M_{200}>3 \times 10^{15} M_{\odot}$ (filled dots).

for $N_{\text {gal }} \equiv N_{\text {spec. }}$. The two estimates of the halo occupation number $\gamma$ are consistent, while the different normalizations reflect the incompleteness of the spectroscopic samples (see Popesso et al. 2006). The orthogonal scatter in both relations is $35 \%$, and $M_{200}$ can be predicted from $N_{\mathrm{gal}}$ with an accuracy of $55 \%$.

Had we not corrected $N_{\text {gal }}$ for the projection effects, we would have underestimated the slope for the $N_{\mathrm{gal}}-M_{200}$ relation, obtaining $0.79 \pm 0.04$. Clearly, applying an average, mass independent, correction to the observed value of $N_{\text {gal }}$ and $L_{\text {op }}$ leads to underestimate the slope of the considered relations.

We check also if different cluster selection techniques introduce biases in our analysis. For this purpose we perform the same analysis separately on the optically and X-ray selected cluster samples, respectively. The observed best fit values are consistent within the statistical errors. Moreover, we perform the analysis by adopting different magnitude cuts to check for vatiation of the Halo Occupation number in different magnitude regimes. We consider the following magnitude cuts: -20 , -17 and -16 mag in the $i$ band. While the normalization of the relation is obviously changing, the best fit values of the Halo Occupation number are cosistent within the errors in all the magniture ranges.

The Halo Occupation Number $\gamma$ has been measured with several different methods in the literature. Most of these come from assuming a form of the HOD, and adjusting the parameters until the prediction from the halo model matches the observed galaxy clustering (e.g. Seljak et al. 2004; Peacock \& Smith 2000; Yang et al. 2003; Zehavi et al. 2004; Magliocchetti \& Porciani 2003). Pisani et al. (2003) used the velocity dispersion in the groups of the Zwicky catalog and obtained $\gamma=0.70 \pm 0.04$, while Marinoni \& Hudson (2002) used the LF of the Nearby Optical Galaxy sample and obtained $\gamma=0.55 \pm 0.043$. 

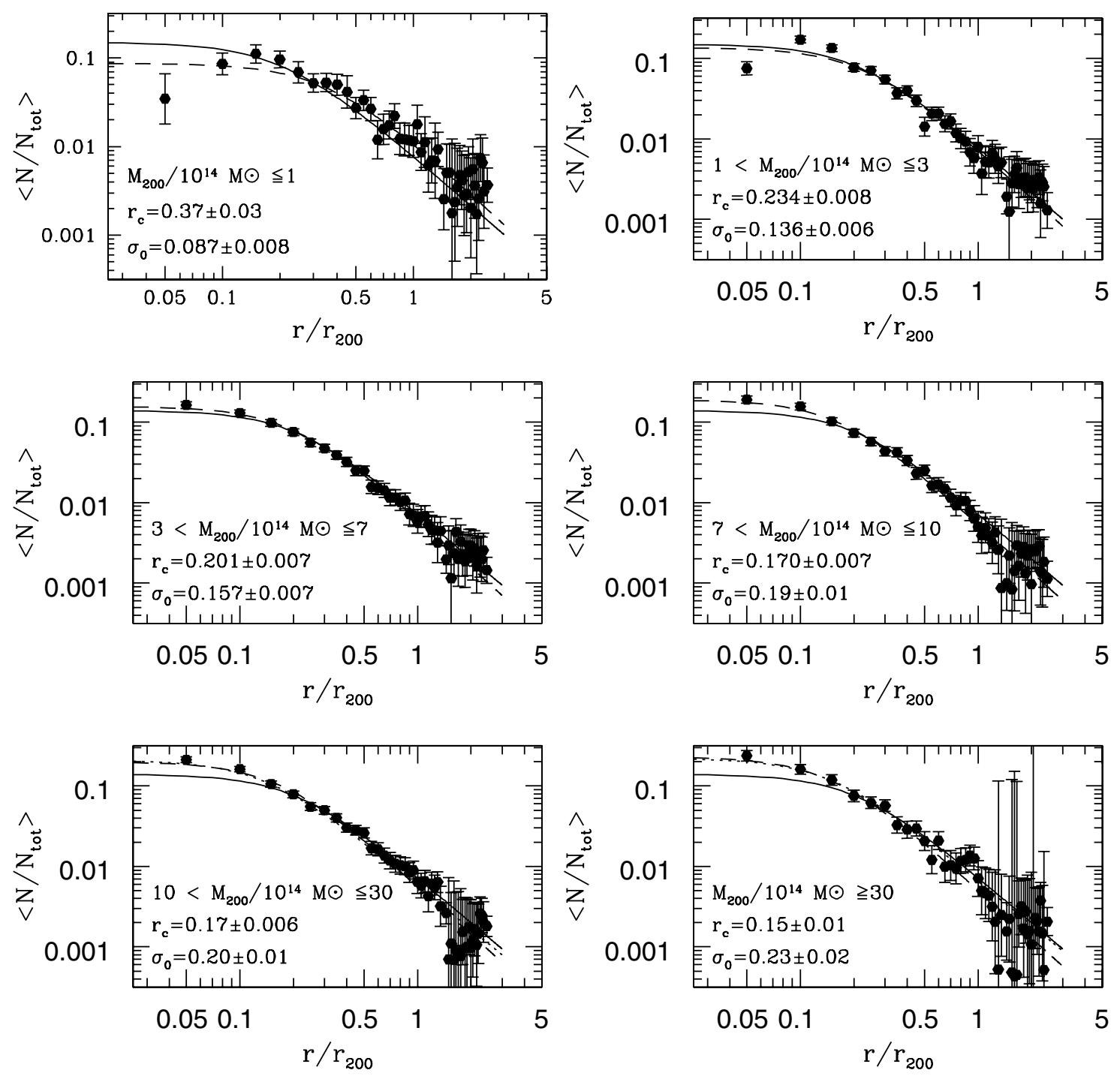

Fig. 7. The stacked surface number density profile of the red cluster galaxies with magnitude $r<-18.5$, separately for clusters of different masses. The meaning of the lines is the same as in Fig. 4.

Many other works in the literature used an approach similar to ours. Kochanek et al. (2003) used a sample of clusters identified in the 2MASS all sky survey, and obtained $\gamma=1.11 \pm 0.09$ on a sample of 84 clusters. Lin et al. (2004) used a sample of 93 X-ray clusters observed in 2MASS, and found $\gamma=0.84 \pm 0.04$. Similar results were obtained by Yang et al. (2005) who used a large sample of groups identified in the 2-degree Field Galaxy Redshift Survey.

With the exclusion of Kochanek et al. (2003), all other studies agree on the fact that the exponent in the $N-M$ relation, and consequently in the $L-M$ relation, is not consistent with unity (see Lin et al. 2004, for a discussion about the discrepancy with the results of Kochanek et al. 2003). However, with the massdependent correction applied to our clusters to clean the $N-M$ $(L-M)$ relation from projection effects, the estimated value of $\gamma$ becomes closer to unity. Nevertheless, a direct proportionality between cluster mass and number of cluster galaxies is still excluded by our analysis at the $\sim 2-2.5 \sigma$ level.

\section{Luminosity function shape and cluster mass}

In this section we investigate whether the lack of galaxies observed in the high-mass systems is related to a different shape of the LFs of clusters of different masses. The universality of the cluster LF has been analysed in two papers of the RASS-SDSS Galaxy Cluster Survey Series (Popesso et al. 2005a, 2006). When measured within the cluster virial radius $\left(r_{200}\right)$, the shape of the LF does not change from cluster to cluster both at the faint and at the bright end (Popesso et al. 2006). Moreover, we observed that the cluster to cluster variations of the LF found in the literature are due to choice of a fixed metric apertures for all the systems. This is due to the fact that fraction of dwarf galaxies in clusters is an increasing function of the clustercentric distance (see also Durret et al. 2002). To keep under control also the possible dependence between the shape of the LF and the cluster mass, we divided our cluster sample (with mass ranges from $10^{13} M_{\odot}$ to $\left.4 \times 10^{15} M_{\odot}\right)$ in three mass bins $\left(M_{200} / 10^{14} M_{\odot} \leq 1\right.$, $1<M_{200} / 10^{14} M_{\odot} \leq 10$ and $\left.M_{200} / 10^{14} M_{\odot}>10\right)$. To increase 

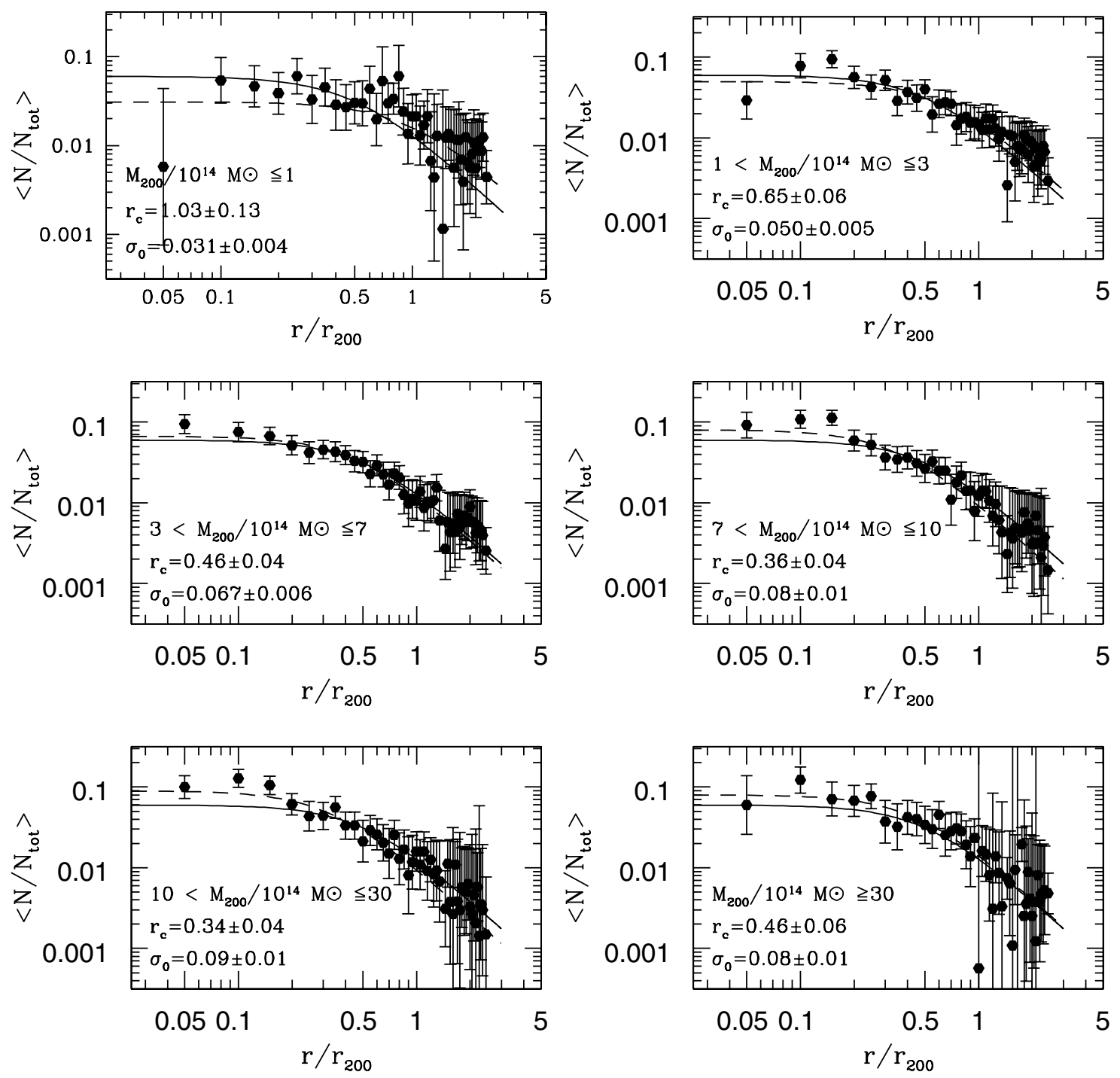

Fig. 8. The stacked surface number density profile of the blue cluster galaxies with magnitude $r<-18.5$, separately for clusters of different masses. The meaning of the lines is the same as in Fig. 4.

the statistics and study the average luminosity distribution of the galaxies in each mass bin, we have used the SDSS photometric data to compute a composite luminosity function (LF) by stacking the individual cluster LFs calculated within $r_{200}$. The individual LFs are obtained by subtracting the field number counts calculated within an annulus around the cluster ( $0.2 \mathrm{deg}$ with), from the number counts in the cluster region, as described in Popesso et al. (2005a). Following previous works, we exclude from the individual cluster LFs the Brightest Cluster Galaxies (BCGs). The composite LF in each mass bin is calculated by following the prescriptions of Colless (1989; see also Popesso et al. 2005a for more details about this method). We require at least 10 clusters contributing to each magnitude bin of the composite LF. This requirement is fulfilled at magnitudes brighter than the absolute magnitude $\operatorname{limit} i-5 \log (h) \leq-16.7$ mag in all the cluster mass bins considered, while $95 \%$ of our clusters have magnitude limits brighter than -18.25 mag in the $i$ band. Thus, we consider galaxies down to 5.5 mag fainter than the cluster $M^{*}$ in this SDSS band (Popesso et al. 2006). Moreover, we use the stellar masses estimated by Kauffmann et al. (2003) for the DR2 spectroscopic sample to evaluate the stellar mass range sampled within this magnitude limit. As shown in Fig. 11, although the scatter is large $(0.18 \mathrm{dex})$, the magnitude cut at -16.7 mag corresponds roughly to a galaxy stellar mass of $1.5 \times 10^{8} M_{\odot}$. As in Sect. 3.3, we distinguish between early and late type galaxies using a SDSS color cut at $u-r=2.22$. In Fig. 12 we show the composite LF for the whole (left panel) and the red (right panel) cluster galaxies populations. In both panel the filled points are the low-mass clusters $\left(M_{200} / 10^{14} M_{\odot} \leq 1\right)$, the empty squares the intermediate-mass systems $\left(1<M_{200} / 10^{14} M_{\odot} \leq 10\right)$ and the empty triangles are the high-mass clusters $\left(M_{200} / 10^{14} M_{\odot}>\right.$ 10). The different mass-bin LFs are renormalized so as to ease the comparisons among them. The solid lines in the panels are the best fits obtained in Popesso et al. (2006) from the X-ray selected RASS-SDSS galaxy clusters for the corresponding whole and red cluster galaxy populations. From Fig. 12 it is clear that, 


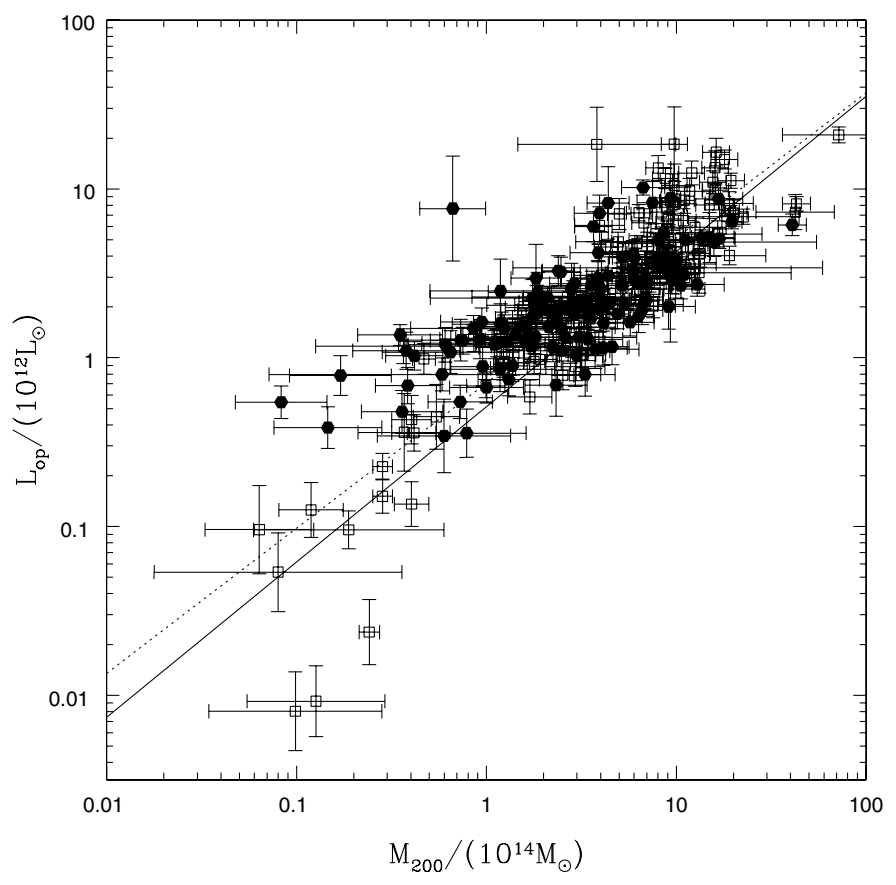

Fig. 9. $L_{\mathrm{op}}-M_{200}$ relation. The optical luminosity is calculated within $r_{200}$ and is corrected for contamination due to projection effect. The empty squares in the plot are the X-ray selected clusters. The filled points are the optically selected clusters. The solid line in the plot is the best fit line of the corrected $L_{\mathrm{op}}-M_{200}$ relation. The dashed line is the best fit line of the uncorrected $L_{\mathrm{op}}-M_{200}$ relation.

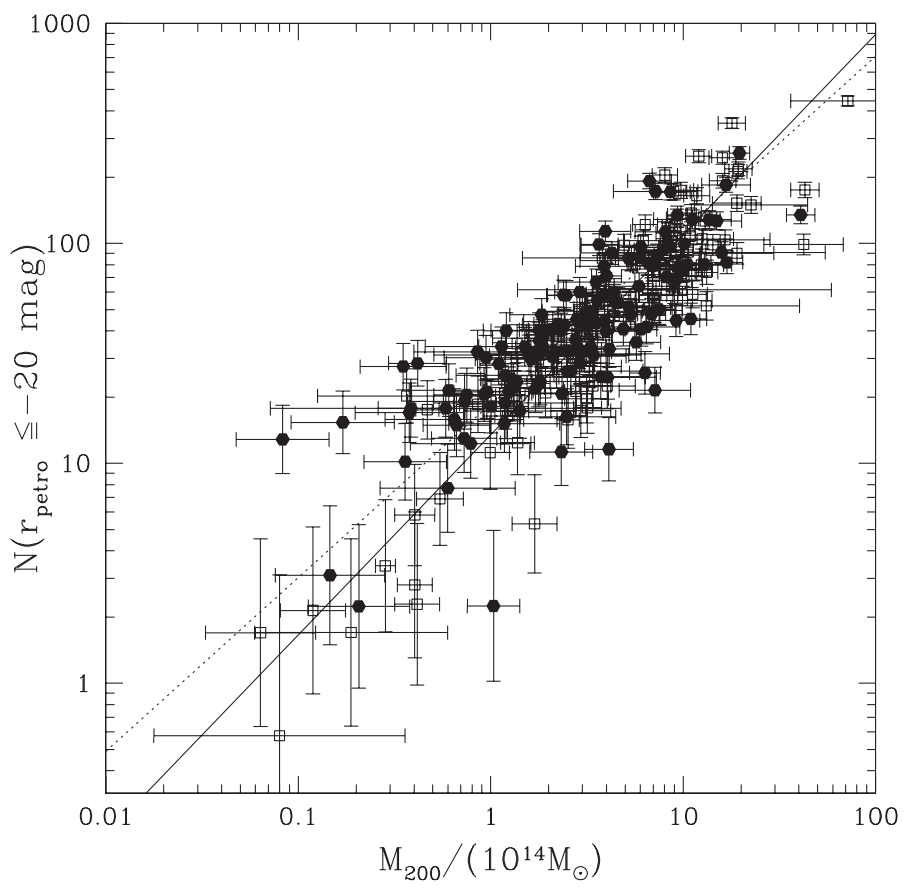

Fig. 10. $N_{\text {gal }}-M_{200}$ relation. The number of cluster galaxies is calculated within $r_{200}$ and is corrected for contamination due to projection effect. The empty squares in the plot are the X-ray selected clusters. The filled points are the optically selected clusters. The solid line in the plot is the best fit line of the corrected $N_{\text {gal }}-M_{200}$ relation. The dashed line is the best fit line of the uncorrected $N_{\text {gal }}-M_{200}$ relation.

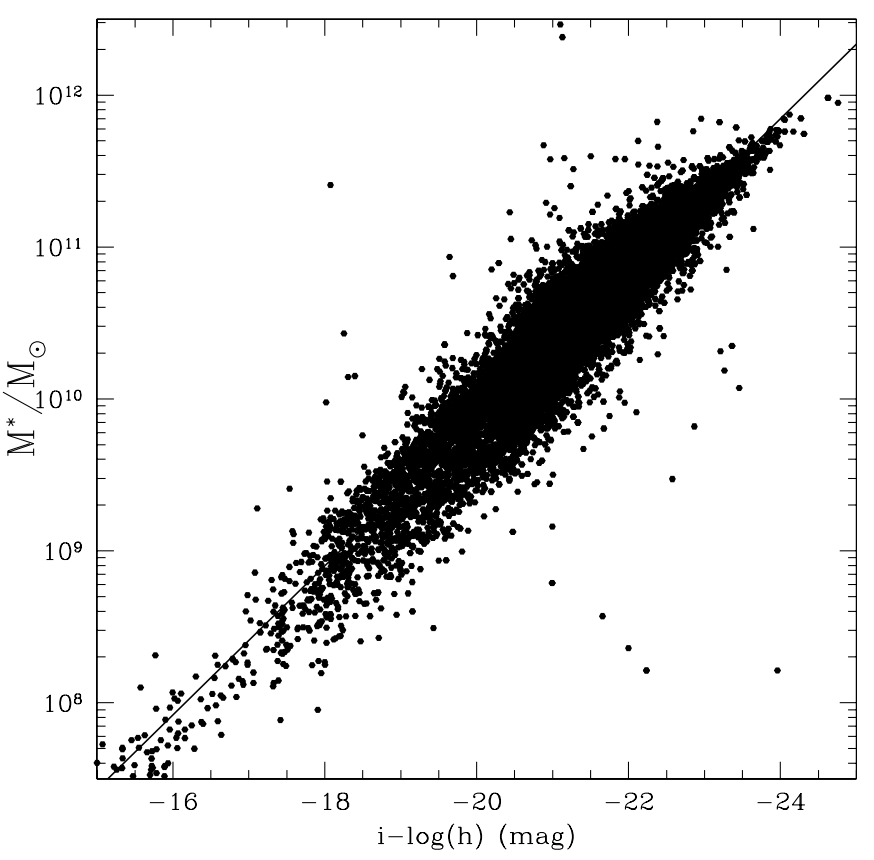

Fig. 11. Relation between the $i$ band Petrosian absolute magnitude and the galaxy stellar mass. The galaxy stellar masses are taken by Kauffmann et al. (2003).

at magnitudes brither than -16.7 mag (alternatively, for galaxy stellar masses above $1.5 \times 10^{8} M_{\odot}$ ), there are no significant differences among the LFs in the different mass bins. Moreover, the best fit of the composite LF of the X-ray selected RASS-SDSS sample provides a very good fit to any of the considered LFs. We conclude that the cluster LF does not depend on the cluster mass. This conclusion is consistent with our previous findings (Popesso et al. 2006).

The previous analysis is based on LFs with the BCGs excluded. Here we examine to what extent can the BCG luminosities be considered the high-end tail extension of the overall cluster LFs. This has been shown not to be the case by previous investigations (e.g. Schechter 1976; Bhavsar \& Barrow 1985). The Schechter function was generally found to provide a good fit to the observed galaxy magnitude distribution as long as the very brightest galaxies, the $\mathrm{cD}$ galaxies, were excluded from the fit (Schechter 1976). The exceptional luminosities of these galaxies have often been interpreted as arising from special processes that are not common to all galaxies, and are particularly effective at the bottom of cluster potential wells. Nevertheless, Lugger (1986) did not find significantly different best-fits to the observed cluster galaxy LFs using Schechter functions, when BCGs were or were not included in the sample.

Following Colless (1989), we normalize the cluster LFs to the number of cluster galaxies in a common magnitude region ( $r \leq-18.5 \mathrm{mag}$ in the present case, see Popesso et al. 2005a for details). Given the number of cluster galaxies in that magnitude region and the best-fit Schechter function of the composite LF, it is possible to calculate the magnitude $M_{r}$ of the $n$th brightest cluster member as the magnitude corresponding to $N\left(M_{r}\right)=n$, where $N\left(M_{r}\right)$ is the analytical form of the cluster integral LF. For this we use the best-fit obtained with a composite of two Schechter functions, after excluding the BCGs. 

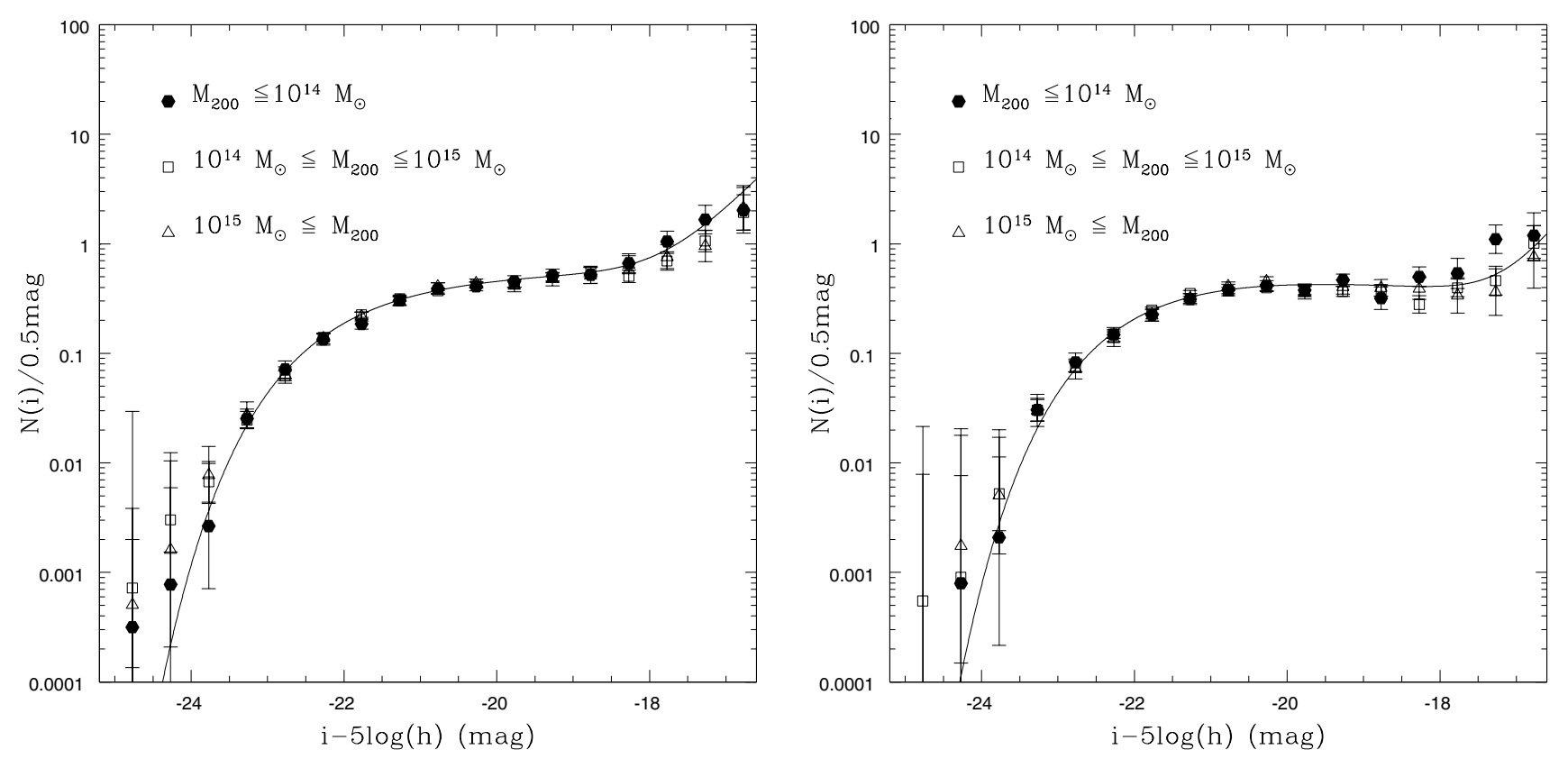

Fig. 12. The composite LF for the whole (left panel) and the red (right panel) cluster galaxy populations. In both panels the filled points are the low-mass clusters $\left(M_{200} / 10^{14} M_{\odot} \leq 1\right)$, the empty squares the intermediate-mass systems $\left(1 \leq M_{200} / 10^{14} M_{\odot} \leq 10\right)$ and the empty triangles are the high-mass clusters $\left(M_{200} / 10^{14} M_{\odot} \geq 10\right)$. The different mass-bin LFs are renormalized so as to ease the comparisons among them. The solid lines in the panels are the best fits obtained in Popesso et al. (2006) from the X-ray selected RASS-SDSS galaxy clusters for the corresponding whole and red cluster galaxy populations.

The left panel of Fig. 13 shows the magnitude of the brightest spectroscopically-confirmed cluster members within $r_{200}$, as a function of the number of cluster galaxies within $r_{200}$ and with $r \leq-18.5 \mathrm{mag}$. The solid line shows the expected magnitude of the brightest galaxies, as estimated from the best-fit LF, vs. the cluster normalization. The dashed line are the statistical uncertainties in the location of the brightest cluster member. Clearly, the estimated magnitudes of the 1st ranked galaxies are consistent with the observed values, as can be judged by considering the median of the 1st-ranked galaxy magnitudes per $N_{\text {gal }}$ bin (filled squares in the plot), and by the fact that $95 \%$ of the BCGs lie within the statistical uncertainties of the expected relation. The middle and the right panel of Fig. 13 are similar to the left panel, but for the 2nd and 3rd brightest cluster galaxies respectively. Again, the agreement between the expected and observed magnitudes is extremely good, and the similarity of these three plots argues against the BCG magnitudes being an anomaly of the cluster LF.

The reason why our result disagrees with previous findings (Postman \& Lauer 1995) must be related to the use of a double (instead of a single) Schechter function for the fit of the observed LF, which allows a better representation of the LF bright end. This was first pointed out by Biviano et al. (1995) in their study of the Coma cluster LF (see also Thompson \& Gregory 1993). The deviation of the cluster LF from a single Schecter function was also found in the clusters extracted from the $N$-body simulations combined with semi-analytical models analysed by Diaferio et al. (1999). They interpreted the LF shape as the effect of the large merger cross-section of the bright and massive central galaxies.

Our result is in agreement with the recent findings of Lin \& Mohr (2004) and Yang et al. (2005) of a tight correlation between the BCG luminosity and the cluster mass. In particular, in the mass range $10^{13} \leq M_{200} / M_{\odot} \leq 10^{15}, L_{\mathrm{BCG}} \propto M_{200}^{0.25}$. The excellent agreement between Lin \& Mohr's result and ours is demonstrated in Fig. 14. There we show the relation between the BCG luminosity and the cluster mass of our cluster sample, where we transformed the cluster $N_{\mathrm{gal}}$ into cluster masses using the HOD we derived in Sect. 4. The solid line in the plot is the best-fit obtained with an orthogonal linear regression, $L_{\mathrm{BCG}} \propto M_{200}^{0.33 \pm 0.04}$, and it is in excellent agreement with the Lin $\&$ Mohr (2004) relation (the dashed line in the plot).

\section{The fundamental plane of cluster ellipticals}

The elliptical galaxies are the dominant population in clusters and therefore any variation of their mass-to-light ratio as a function of the cluster mass could contribute to affecting the slope of the $N-M$ and the $L-M$ relations. Thus, one could still have a constant ratio between the total cluster mass and the total mass in galaxies, even for $\gamma<1$ (see Sect. 4), if galaxies of given luminosity have higher masses in higher-mass clusters.

To investigate whether elliptical galaxies in high mass clusters have a higher average $M / L$ than their counterparts in low mass systems, we determine the fundamental plane (FP hereafter) of ellipticals as traced by the spectroscopic members of each cluster within $r_{200}$. The FP relates the effective radius of the luminosity distribution of ellipticals, $r_{\mathrm{e}}$, with their internal velocity dispersion, $\sigma$, and their surface brightness (Djorgovsky \& Davis 1987). If the virial radius of ellipticals is linearly proportional to $r_{\mathrm{e}}$ and their internal velocity dispersion to the virial value, the FP effectively can be used to constrain the mass-tolight ratio of elliptical galaxies.

For this analysis, as before, we have divided our cluster sample in three subsamples of low-, intermediate-, and high-mass. Ellipticals are identified within each cluster using the selection 

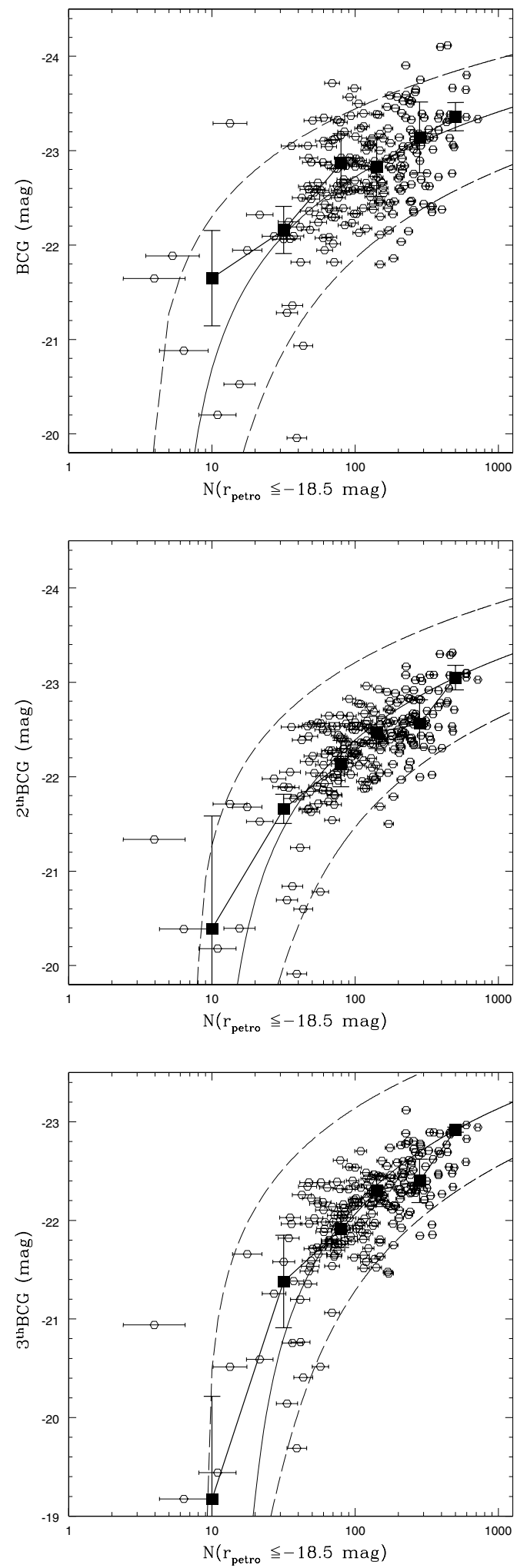

Fig. 13. Upper panel: the magnitude of the BCGs (empty squares) within $r_{200}$ as a function of the number of cluster galaxies within the same radius, and with $r \leq-18.5 \mathrm{mag}$. The solid line in the plot shows the expected BCG magnitude as estimated from the best-fitting composite LF, as a function of the cluster normalization. The dashed lines are the statistical uncertainties in the relation represented by the solid line. The filled squares are the median magnitudes per bin of $N_{\mathrm{gal}}$. Middle panel: same as the left panel, but for the second brightest cluster galaxies. Bottom panel: same as the left panel, but for the third brightest cluster galaxies.

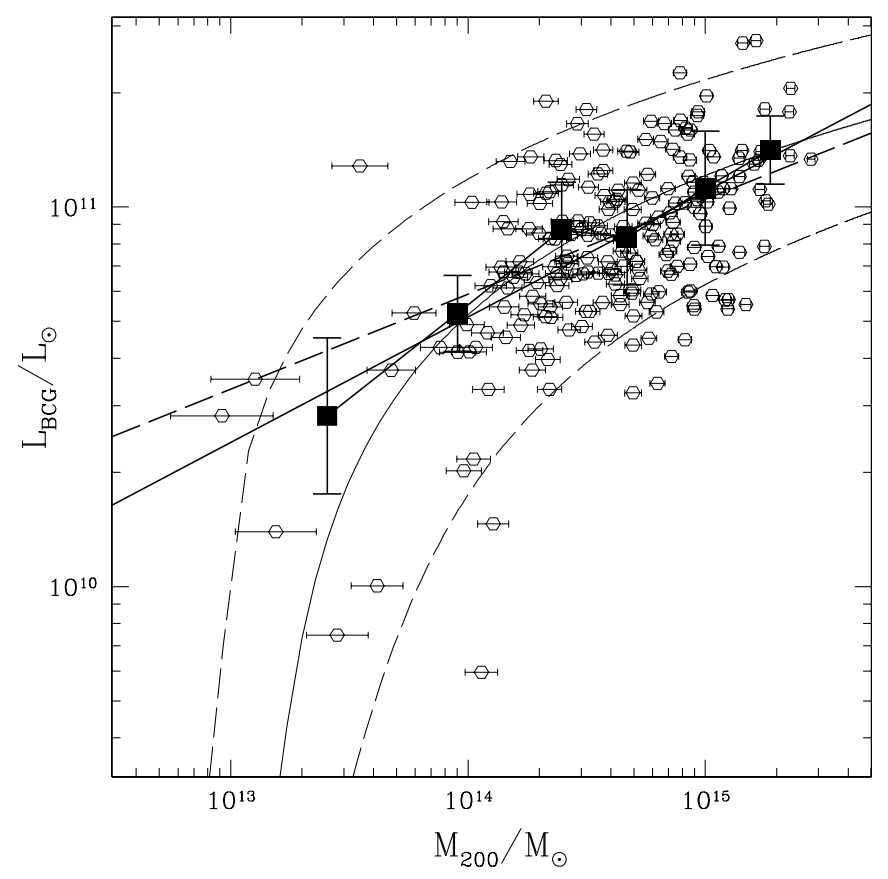

Fig. 14. The relation between BCG luminosity and cluster mass for our cluster sample. Cluster masses are computed from $N_{\text {gal }}$ s using our derived HOD. The solid line in the plot is the best-fit line obtained with an orthogonal linear regression, $L_{\mathrm{BCG}} \propto M_{200}^{0.33 \pm 0.04}$, and the dashed line is Lin \& Mohr's (2004) relation. Other symbols have the same meaning as in the Fig. 13.

criteria of Bernardi et al. (2003a). As a measure of the effective radius we use the Petrosian radius $r_{50}$, which encloses $50 \%$ of the total Petrosian luminosity, multiplied by the square root of the ratio $b / a$ of the lengths of the minor and major axes of the observed surface brightness profile. The SDSS spectroscopic catalog contains a measure of the line of sight velocity dispersion which has been corrected for aperture effects as in Bernardi et al. (2003a). In what follows, we show the best correlation between the variables $r_{\mathrm{e}}, \sigma$ and $\mu=-2.5 \log \left[(L / 2) / r_{\mathrm{e}}^{2}\right]$ in the SDSS $r$-band. Data are fitted with the ODRPACK routine (Akritas \& Bershady 1996).

The left-hand side panel of Fig. 15 shows the best-fit FP relating the three variables $\sigma, r_{\mathrm{e}}$ and $\mu$; our result is consistent with Bernardi et al. (2003b). The different symbols in the plot indicate elliptical galaxies of clusters in different mass bins. We do not find any variation of the best-fit FP for the different mass samples. In the right panel of the same figure we show the mean residuals from the FP of the elliptical populations of individual clusters as a function of the cluster masses. The scatter around the FP is $\sim 10 \%$ as in Bernardi et al. (2003b) and the mean residual is consistent with zero independent of the cluster mass.

Any variation of the average mass-to-light ratio of the ellipticals would result in a variation of their FP. The constancy of the FP across the cluster mass range therfore implies a constant mass-to-light ratio of the cluster ellipticals.

\section{Discussion}

Using a large sample of galaxy clusters we have shown that the number of galaxies per unit mass is lower in clusters of higher masses, i.e. the slope of the relation $N \propto M^{\gamma}$ is $\gamma<1$ at the $2.5 \sigma$ 

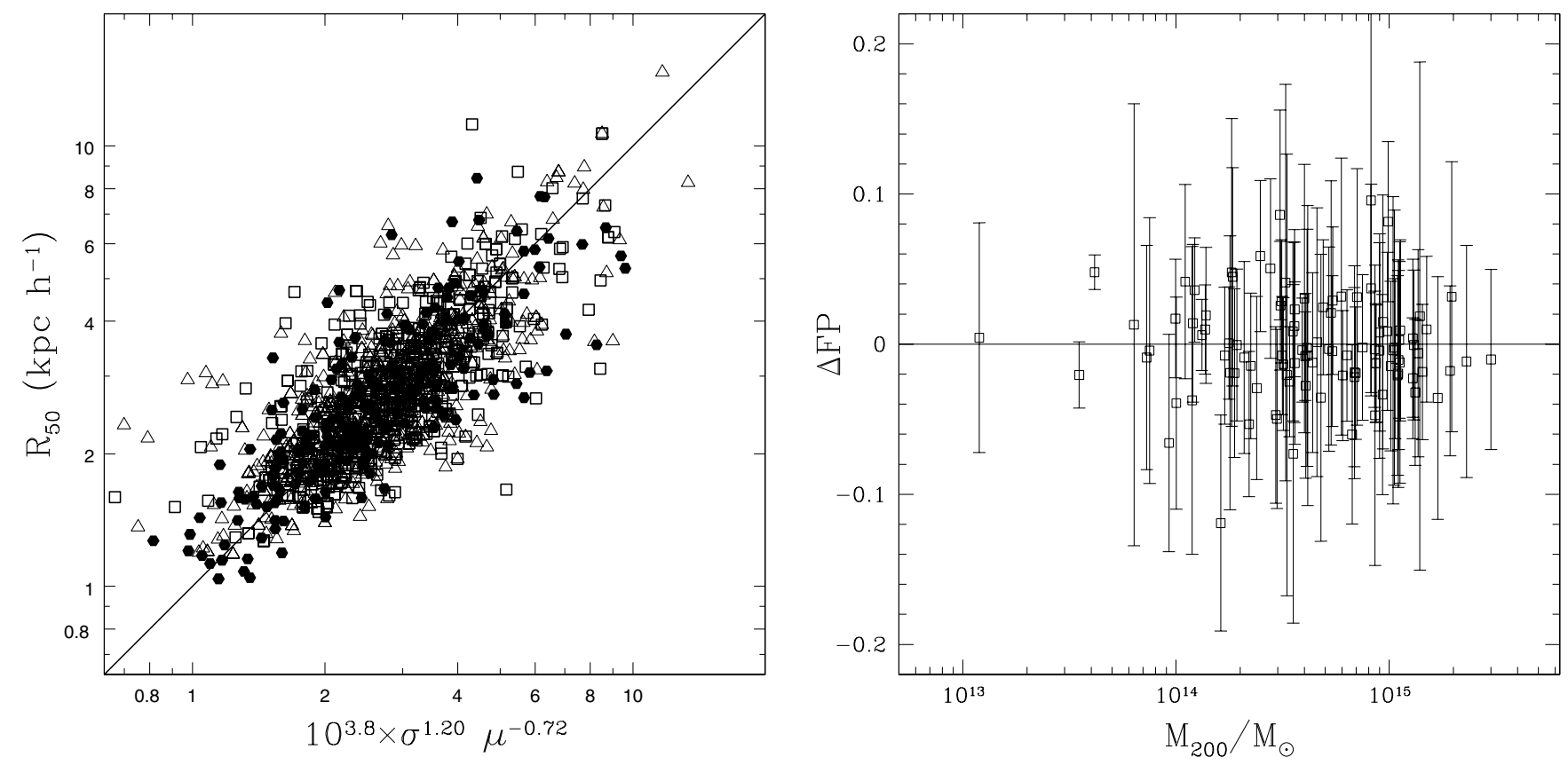

Fig. 15. Left panel: the best-fit FP of cluster ellipticals, relating their internal velocity dispersions $(\sigma)$, effective radii $\left(r_{\mathrm{e}}\right)$, and surface brightnesses within $r_{\mathrm{e}}(\mu)$. The different symbols in the plot indicate elliptical galaxies of clusters in different mass bins. The right-hand side panel shows the mean residuals from the global FP of the elliptical populations of individual clusters as a function of the cluster mass.

level. Our result is thus in agreement with previous findings (see, e.g., Lin et al. 2004, and references therein) although we find a somewhat steeper $N-M$ relation ( $\gamma$ closer to unity), because of our mass-dependent correction for projection effects.

From the theoretical point of view, $\gamma<1$ is expected. On one hand, hierarchical models of structure formation predict a universal mass distributions of the subhalos (see, e.g., De Lucia et al. 2004; and Gao et al. 2004), independent of the mass of the parent halo. As a consequence, the number of subhalos is directly proportional to the parent halo mass $(N \propto M)$. On the other hand, including baryons in the simulations leads to a decreasing number of galaxies per given mass in halos of higher masses (i.e. $\gamma<1$, see, e.g., Benson et al. 2000a,b; White et al. 2001; Berlind et al. 2003), and of an increasing $M / L$ as a function of mass (e.g. Kauffmann et al. 1999). This could happen as the consequence of a decreasing efficiency of gas cooling and star formation (see, e.g., Springel \& Hernquist 2003; Berlind et al. 2003; Borgani et al. 2004; Kravtsov et al. 2004), or because of an increased merger rate (White et al. 2001), or of an increased destruction rate of galaxies (Lin et al. 2003), as the mass of the parent halo increases.

Although we observe $\gamma<1$ as predicted, a deeper look at other properties of our clusters seems to be at odds with theoretical predictions. If mergers and/or tidal effects are responsible for reducing the number of galaxies per given mass in clusters of higher masses, we expect to see these processes to leave an imprint into the cluster LFs. Instead, we find that the LF is universal, with no dependence on the cluster mass. Our result is at odds with Lin et al. (2004). The reason for this difference is unclear, but it could be related to the different photometric bands (Lin et al. use the $K$-band), although it is difficult to see why the LFs of clusters of different masses should look identical in four SDSS photometric bands and different in the $K$-band.
Another result argues against galaxy-destruction via tidal stripping being more efficient in higher mass clusters. Higher mass clusters are characterized by a more concentrated number density profile (see Sect. 3.3) and a less concentrated mass density profile (NFW, Katgert et al. 2004; Pratt \& Arnaud 2005) near the centre. If anything, this is consistent with a picture where galaxies are more likely to survive near the centre of higher mass clusters, while galaxies in lower-mass clusters are destroyed when crossing the cluster core, because of the efficient tidal stripping resulting from a more concentrated halo mass profile.

A lower efficiency of star formation in galaxies of higher mass clusters would also lead to observing $\gamma<1$ in the HOD. A consequence of this process should be visible in a decreased $M / L$ for the galaxies of higher mass clusters, as compared to the galaxies of lower mass clusters. We have explored this possibility by the analysis of the FP of cluster ellipticals. No evidence for a variation of the FP as a function of cluster mass was found. This result argues for a constant $M / L$ and hence a similar star formation efficiency of cluster ellipticals in clusters of different masses, in agreement with the predictions of Diaferio et al. (2001), based on numerical simulations combined with semi-analytical models of galaxy formation. Note, however, that Springel et al. (2001) have argued that even heavily stripped cluster galaxies obey the Faber-Jackson relation, since the internal velocity dispersion of a stripped subhalo remains relatively stable until it is fully disrupted. Hence the constancy of the FP does not rule out the possibility of subhalos stripping.

Bahcall \& Comerford (2002) have suggested that the observed increasing $M / L$ of clusters as a function of cluster masses is a consequence of a higher fraction of galaxies with evolved stellar populations in higher mass clusters. There is no evidence for this in our data (Popesso et al. 2005e). Moreover, Bahcall $\&$ Comerford's prediction that $M / L$ vs. $M$ would become flatter 
when the photometric band is moved to longer wavelengths, is also ruled out by our data, where we see that the relation does not change by changing the SDSS photometric band, in agreement with the results of numerical simulations combined with semi-analytical modelling (Kauffmann et al. 1999).

How can we, then, reconcile the observed $N-M$ with the predictions for a universal subhalo mass distribution? It is hard to find physical processes capable of reducing the number of observed galaxies per given mass, while at the same time leaving the subhalo mass distribution, the galaxy LF, and the average galaxy mass-to-light ratios unchanged. Hence, the most likely explanation is that the mass distribution of the subhalos is not universal and the observed $\gamma<1$ for galaxies simply reflect an underlying $\gamma<1$ for subhalos.

Our correction for projection effects does work in the sense of changing the observed $\gamma$ of the $N \propto M^{\gamma}$ closer to unity. The resulting $\gamma$ is still found to be below unity, but the significance of this is not overwhelming $(2.5 \sigma$ level $)$. Hence it is well possible that other insofar unapplied corrections, or, perhaps, an improved correction for the projection effects, could make $\gamma$ consistent with unity, thus reconciling theory and observations.

\section{Conclusions}

We have studied the $L-M$ and the $N-M$ relations in the 4 SDSS bands $g, r, i, z$ for a sample of 217 galaxy clusters with confirmed 3D overdensity in the SDSS DR3 spectroscopic catalog. All the quantities are measured within the characteristic cluster radius $r_{200}$. We have remarked upon the direct connection between the two relations due to the proportionality of the cluster optical luminosity and the number of cluster galaxies.

We have studied the galaxy surface number density profile in five bins of cluster mass and discovered that the profile has a strong dependence on the cluster mass. In the low and intermediate mass systems the best fit is provided by a King profile. The core radius of the best fit decreases as a function of the cluster mass, while the central galaxy density increases. In the highest mass bins a more concentrated generalized King profile or a cuspy NFW profile provide the best fits. Using the best fit profile in each mass bin, we have converted the observed number of cluster galaxies to the value within the virial sphere. Since clusters of different masses exhibit different surface density profiles, the deprojection correction decreases with the cluster mass. Applying this mass-dependent correction affects the $L-M$ and $N-M$ relations, by increasing the slope of these relations to the value of $0.92 \pm 0.03$. Similarly, the slope of the $M / L-M$ relation also is affected and becomes $0.18 \pm 0.04$. Hence, neglecting the dependence of the deprojection correction on the cluster mass leads one to underestimate the slope of the $L_{\mathrm{op}}-M_{200}$ and $N_{\text {gal }}-M_{200}$ relations. Despite the deprojection correction, the derived $N-M$ and the $L-M$ relations are still only marginally consistent with unity, at the $2.5 \sigma$ level, i.e. direct proportionality between cluster mass and number of cluster galaxies is not supported.

We have compared the properties of our clusters with the prediction of the hierarchical models of structure formation. These models naturally predict that $N \propto M^{\gamma}$ with $\gamma<1$. This result is generally interpreted as an indication that the galaxies in the low mass systems are older and more luminous per unit mass than the galaxies in high mass clusters. As a consequence, variations of the shape of the cluster LF and of the elliptical FP with the cluster mass are also expected. Such predicted variations are however not seen in our data. Not only we have found the LF to be the same for clusters of different masses, but we also proved that this universal LF can be used to accurately predict the magnitudes of the three brightest cluster galaxies, given the LF-normalization of the clusters in which they are located. In other words, the BCG magnitudes are consistent with being drawn from the best-fit magnitude distribution of other cluster galaxies. Moreover we have shown that the FP of cluster ellipticals has the same slope in all the clusters and does not depend on the cluster mass.

From the observational point of view, the mean cluster luminosity function and the $N-M$ or the $L-M$ relation determine completely the luminosity distribution of cluster galaxies. The mean cluster LF constrains with high accuracy the shape of the luminosity distribution in clusters, while the $N-M$ relation, calculated in a given magnitude range, fixes the normalization of the LF as a function of the cluster mass. Forthcoming cosmological models of galaxy formation should aim at reproducing this characteristic of the cluster galaxy population, in order to understand the processes of galaxy formation and evolution in the cluster enviroment.

Acknowledgements. We thank the referee, Christophe Adami, for the useful comments which helped in improving the paper. We acknowledge useful discussions with Stefano Borgani and Simon White. Funding for the creation and distribution of the SDSS Archive has been provided by the Alfred P. Sloan Foundation, the Participating Institutions, the National Aeronautics and Space Administration, the National Science Foundation, the US Despartment of Energy, the Japanese Monbukagakusho, and the Max Planck Society. The SDSS Web site is http://www.sdss.org/. The SDSS is managed by the Astrophysical Research Consortium (ARC) for the Participating Institutions. The Participating Institutions are The University of Chicago, Fermilab, the Institute for Advanced Study, the Japan Participation Group, The Johns Hopkins University, Los Alamos National Laboratory, the Max-Planck-Institute for Astronomy (MPIA), the Max-Planck-Institute for Astrophysics (MPA), New Mexico State University, University of Pittsburgh, Princeton University, the United States Naval Observatory, and the University of Washington.

\section{References}

Abazajian, K., Adelman, J., Agueros, M., et al. 2003, AJ, 126, 2081 (Data Release One)

Adami, C., Mazure, A., Biviano A., Katgert, P., \& Rhee, G. 1998a, A\&A, 331, 493

Adami, C., Mazure, A., Katgert, P., \& Biviano A. 1998b, A\&A, 336, 63

Adami, C., Mazure, A., Ulmer, M. P., Savine, C. 2001, A\&A, 371, 11

Akritas, M. G., \& Bershady, M. A. 1996, ApJ, 470, 706

Bahcall, N. A., \& Comerford, J. M. 2002, ApJ, 565, L5

Bartelmann, M. 1996, A\&A, 313, 697

Beers, T. C., Flynn, K., \& Gebhardt 1990, AJ, 100, 32

Benson, A. J., Baugh, C. M., Cole, S., Frenk, C. S., \& Lacey, C. G. 2000a, MNRAS, 316, 107

Benson, A. J., Cole, S., Frenk, C. S., Baugh, C. M., \& Lacey, C. G. 2000b, MNRAS, 311, 793

Berlind, A. A., \& Weinberg, D. H. 2002, ApJ, 575, 587

Berlind, A. A., Weinberg, D. H., Benson, A. J., et al. 2003, ApJ, 593, 1

Bernardi, M., Sheth, R. K., Annis, J., et al. 2003a, AJ, 125, 1817

Bernardi, M., Sheth, R. K., Annis, J., et al. 2003b, AJ, 125, 1866

Bhavsar S. P., \& Barrow, J. D 1985, MNRAS, 213, 857

Biviano A., Durret F., Gerbal D., et al. 1995, A\&A, 297, 610

Biviano, A., \& Girardi, M. 2003, ApJ, 585, 205

Blanton, M. R., Lupton, R. H., Maley, F. M., et al. 2003, AJ, 125, 2276 (Tiling Algorithm)

Borgani, S., Murante, G., Springel, V., et al. 2004, MNRAS, 348, 1078

Carlberg, R. G., Yee, H. K., \& Ellingson, E. 1997, ApJ, 478, 462

Colless, M. 1989, MNRAS, 237, 799

De Lucia, G., Kauffmann, G., Springel, V., et al. 2004, MNRAS, 348, 333 de Theije, P. A. M., Katgert, P., \& van Kampen, E. 1995, MNRAS, 273, 30 Diaferio, A., Kauffmann, G., Balogh, M. L., et al. 2001, MNRAS, 323, 999 Diaferio, A., Kauffmann, G., Colberg, J. M., \& White, S. D. M. 1999, MNRAS, 307,537

Dolag, K., Bartelmann, M., Perrotta, F., et al. 2004, A\&A, 416, 853

Djorgovski S., \& Davis M. 1987, ApJ, 313, 59 
Durret, F., Adami, C., \& Lobo, C. 2002, A\&A, 393, 439

Eisenstein, D. J., Annis, J., Gunn, J. E., et al. 2001, AJ, 122, 2267

Fasano, G., Pisani, A., Vio, R., \& Girardi, M. 1993, ApJ, 416, 546

Fukugita, M., Ichikawa, T., \& Gunn, J. E. 1996, AJ, 111, 1748

Gao, L., White, S. D. M., Jenkins, A., et al. 2004, MNRAS, 355, 819

Girardi, M., Biviano, A., Giuricin, G., et al. 1995, ApJ, 438, 527

Girardi, M., Giuricin, G., Mardirossian F., et al. 1998, ApJ, 505, 74

Girardi, M., Manzato, P., Mezzetti, M., et al. 2002, ApJ, 569, 720

Gunn, J. E., Carr, M. A., Rockosi, C. M., et al 1998, AJ, 116, 3040 (SDSS Camera)

Hogg, D. W., Finkbeiner, D. P., Schlegel, D. J., \& Gunn, J. E. 2001, AJ, 122, 2129

Katgert, P., Biviano, A., \& Mazure, A. 2004, ApJ, 600, 657

Kauffmann, G., Nusser, A., \& Steinmetz, M. 1997, MNRAS, 286, 795

Kauffmann, G., Colberg, J. M., Diaferio, A., \& White, Simon D. M. 1999 , MNRAS, 303, 188

Kauffmann, G., Heckman, T. M., White, S. D. M., et al. 2003, MNRAS, 341, 33

King, I. 1962, AJ, 67, 274

Kochanek, C. S., White, M., Huchra, J., et al. 2003, ApJ, 585, 161

Kravtsov, A. V., Berlind, A. A., Wechsler, R. H., et al. 2004, ApJ, 609, 35

Lin, Y.-T., Mohr, J. J., \& Stanford, S. A. 2003, ApJ, 591, 749

Lin Y. T., \& Mohr J. J. 2004, ApJ, 617, 879

Lin Y. T., Mohr J. J., \& Stanford, S. A. 2004, ApJ, 610, 745

Lugger, P. M. 1986, ApJ, 303, 535

Lupton, R. H., Gunn, J. E., \& Szalay, A. S. 1999, AJ, 118, 1406

Lupton, R., Gunn, J. E., Ivezić, Z., et al. 2001, in Astronomical Data Analysis Software and Systems X, ed. F. R. Harnden, Jr., F. A. Primini, \& H. E. Payne (San Francisco: Astr. Soc. Pac.), ASP Conf. Ser., 238, 269 [arXiv: astro-ph/0101420]

Ma, C., \& Fry, J. N. 2000, ApJ, 538, L107

Magliocchetti, M., \& Porciani, C. 2003, MNRAS, 346, 186

Marinoni, C., \& Hudson, M. J 2002, ApJ, 569, 101

Navarro, J. F., Frenk, C. S., \& White, S. D. M. 1996, ApJ, 462, 563

Navarro, J. F., Frenk, C. S., \& White, S. D. M. 1997, ApJ, 490, 493

Oort, J H 1958, in La structure et l'évolution de l'univers, ed. R. Stoopes (Brussels: Solvay Inst), 163

Peacock, J. A., \& Smith, R. E. 2000, MNRAS, 318, 1144

Pisani, A., Ramella, M., \& Geller, M. J. 2003, AJ, 126, 1677
Plionis, M., Basilakos, S., \& Tovmassian, H. M. 2004, MNRAS, 352, 1323 Popesso, P., Böhringer, H., Brinkmann J., et al. 2004, A\&A, 423, 449 (Paper I) Popesso, P., Biviano, A., Böhringer, H., et al. 2005a, A\&A, 433, 415 (Paper II) Popesso, P., Biviano, A., Böhringer, H., \& Romaniello, M. 2005b, A\&A, 433, 431 (Paper III)

Popesso, P., Biviano, A., Böhringer, H., \& Romaniello, M. 2006, A\&A, 445, 29 (Paper IV)

Popesso, P., Biviano, A., Böhringer, H., \& Romaniello, M. 2007, A\&A, 461, 397 (Paper V)

Postman M., \& T. Lauer 1995, ApJ, 440, 28

Pratt G. W., \& Arnaud M. 2005, A\&A, 429, 791

Rines, K., Geller, M. J., Diaferio, A., et al. 2004, AJ, 128, 1078

Ramella, M., Boschin, W., Geller, M. J., Mahdavi, A., \& Rines, K. 2004, AJ, in press [arXiv: astro-ph/0407640]

Sarazin, C. L. 1988, X-Ray Emission from Clusters of Galaxies, British Astron. Assoc. J., 98, 212

Schechter, P. 1976, ApJ, 203, 297

Scoccimarro, R., Sheth, R. K., Hui, L., et al. 2001, ApJ, 546, 20

Seljak, U. 2000, MNRAS, 318, 203

Smith, J. A., Tucker, D. L., Kent, S. M., et al. 2002, AJ, 123, 2121

Springel, V., \& Hernquist, L. 2003, MNRAS, 339, 312

Springel, V., White, S. D. M., Tormen, G., \& Kauffmann, G. 2001, MNRAS, 328,726

Stoughton, C., Lupton, R. H., Bernardi, M., et al. 2002, AJ, 123, 485

Strateva, I., Ivezić, Z., Knapp, G., et al. 2001, AJ, 122, 1861

Strauss, M. A., Weinberg, D. H., Lupton, R. H., et al. 2002, AJ, 124, 1810

Tegmark, M., Strauss, M., Blanton, M., et al. 2004, PhRvD

The L. S., \& White, S. D. M. 1986, AJ, 92, 1248

Thompson, L. A., \& Gregory, S. A. 1993, AJ, 106, 2197

White, M., Hernquist, L., \& Springel, V. 2001, ApJ, 550, 129

Yang, X., Mo, H. J., \& van den Bosch, F. C. 2003, MNRAS, 339, 1057

Yasuda, N., Fukugita, M., Narayanan, V. K., et al. 2001, AJ, 122, 1104

Yoshikawa, K., Taruya, A., Jing, Y. P., \& Suto, Y. 2001, ApJ, 558, 520

York, D. G., Adelman, J., Anderson, J. E., et al. 2000, AJ, 120, 1579

Zehavi, I., Weinberg, D. H., Zheng, Z., et al. 2004, ApJ, 608, 16

Zheng Z., Berlind A. A., Weinberg D. H., et al. 2005

[arXiv: astro-ph/0408564] 